\section{Hair Loss Following Typhoid Fever: A Forgotten Phenomenon}

SIR-Typhoid fever is currently a rare disease in Switzerland, as only 45 cases were reported in 1992. Effective treatments for typhoid fever have become available in the last few decades, and life-threatening salmonellal infections are now rare. Before treatment was available, reversible effluvium was a well-recognized clinical finding during the final stages of typhoid fever [1-4]. In contrast, hair loss has not been reported since the introduction of antimicrobial agents; not only has it not been reported in large clinical trials of typhoid fever $[5,6]$, but it is not even mentioned in modern textbooks on infectious diseases [7]. We recently studied a small epidemic outbreak of Salmonella typhi infections in which all patients experienced symptomatic alopecia, a finding that indicates that this symptom may still be associated with typhoid fever.

An 83-year-old female, the index patient, was admitted to the hospital because of critical impairment of consciousness on day 11 of a high fever. Cultures of blood and stool specimens yielded S. typhi, and, after receiving antibiotic treatment with ciprofloxacin for 14 days, she recovered without any sequelae. Stool specimens were immediately obtained from all 15 persons who lived in the index patient's household or who were employed at the nursery where the patient worked, and these 15 persons were given therapy with oral ciprofloxacin ( 250 or $500 \mathrm{mg}$ b.i.d.). At the time the stool specimens were collected, three of these 15 individuals already had had nonspecific symptoms of infection (e.g., cough, diarrhea, chills, and fever [temperature of $\leqslant 41^{\circ} \mathrm{C}$ ] for 4-10 days); culture of these specimens yielded $S$. typhi.

The probable source of the infection was a 24-year-old male employee from the Middle East who was employed in the nursery where the patient worked; this employee had had typhoid fever a few months before the index patient became ill and had not complied with the prescribed antibiotic therapy. S. typhi isolates from two patients (as well as isolates from the source patient's stool specimens) were available for epidemiologic typing; isolates from all three patients shared lysotype A and PstI ribopattern $E[8]$, indicating that all patients were infected by the same source.

The general conditions of all three patients improved within days of receiving therapy, but $2-4$ weeks (mean \pm SD, $3.6 \pm 0.8$ weeks) after initiation of ciprofloxacin (i.e., 2.5-8 weeks after the onset of symptoms) all patients spontaneously reported diffuse loss of scalp hair (they attributed this loss to the antibacterial therapy). The source patient had also noted effluvium. All 11 uninfected individuals who were receiving ciprofloxacin were contacted, and none reported hair loss. Within 9-16 weeks, a complete reversal of effluvium was noted for the three previously healthy younger patients (24-46 years old), and a partial reversal was noted for the two elderly patients ( 81 and 83

\footnotetext{
Reprints or correspondence: Dr. Walter E. Haefeli, Division of Clinical Pharmacology, Department of Internal Medicine, University Hospital (Kantonsspital), Petersgraben 4, CH-4031 Basel. Switzerland.

Clinical Infectious Diseases 1995;20:723-4

(C) 1995 by The University of Chicago. All rights reserved.

$1058-4838 / 95 / 2003-0048 \$ 02.00$
}

years old) who received long-term maintenance therapy with enalapril and reserpine, respectively, because of mild hypertension. Chronic carriage of $S$. typhi was excluded in all cases on the basis of the (negative) results of follow-up stool cultures: these results were obtained several weeks after all the patients completed antibiotic therapy.

Earlier this century, alopecia was a well-known phenomenon that occurred as a consequence of highly febrile bacterial and viral infections [2-4]. During the 1918 influenza epidemic, in particular, dramatic but reversible effluvium was noted in many cases 1-2.5 months following infection [2, 3]. Effluvium has also been reported to occur as a consequence of bacterial infections such as pneumonia, scarlet fever, and typhoid fever. As postfebrile effluvium usually occurs in patients with fever (temperature of $>39^{\circ} \mathrm{C}$ ) [3], the availability of effective antipyretic and antibiotic treatment may have decreased its incidence. This may explain why we could not find a report of typhoid fever associated with alopecia after extensively searching the world literature with use of on-line media (MEDLINE, Pharmline, IOWA Drug Information, Toxline, EMBASE, PARA, and Health Periodicals).

In our cases, an infectious etiology of effluvium is supported by several facts: (1) Despite the widespread use of ciprofloxacin. there are no reports relating alopecia to this drug and the manufacturer was aware only of five reports in which effluvium occurred during ciprofloxacin therapy and in which the causal relationship between this therapy and effluvium was not confirmed; (2) None of the 11 healthy individuals who received ciprofloxacin had symptomatic effluvium; and (3) The facts that all patients had a prolonged course of disease and developed a high fever before therapy was initiated may have favored the manifestation of effluvium.

We describe a troubling but reversible symptom of febrile salmonellal infections that has been forgotten. The findings of our cases may therefore be valuable for patients who experience effluvium following typhoid fever and for physicians who treat patients worried about hair loss.

Walter E. Haefeli, Rudolf Ott, Andreas J. Bircher, and André P. Burnens

Department of Internal Medicine. Division of Clinical Pharmacologl, and Department of Dernatologl. University: Hospital, Basel; general practice, Biel-Benken; and Swiss Reference Laboratory for Foodborne Diseases. Bern, Switzerland

References

1. Kligman AM. Pathologic dynamics of human hair loss. Arch Dermatol 1961;84:175-98.

2. Richter R. Die Haare. In: Jadassohn J, ed. Handbuch der Haut- und Geschlechtskrankhciten. Ergänzungswerk. Vol 1. Part 3. Berlin: Springer-Verlag, 1963:472.

3. Darier J. Précis de dermatologie. 4th ed. Paris: Masson, 1928:543.

4. Galwesky E. Erkrankungen der Haare und des Haarbodens. In: Jadassohn J, ed. Handbuch der Haut- und Geschlechtskrankheiten. Vol. 13. Berlin: Springer-Verlag, 1932:221

5. Stuart BM. Pullen RL. Typhoid: clinical analysis of three hundred and sixty cases. Arch Intern Med 1946;78:629-61. 
6. Saphra I, Winter JW. Clinical manifestations of salmonellosis in man: an evaluation of 7779 human infections identified at the New York Salmonella Center. N Engl J Med 1957;256:1128-34.

7. Hook EW. Salmonella species (including typhoid fever). In: Mandell GL, Douglas RG Jr, Bennett JE, eds. Principles and practice of in- fectious diseases. 3rd ed. New York: Churchill Livingstone, 1990: 1700-16.

8. Altwegg M. Hickman-Brenner FW, Farmer JJ III. Ribosomal RNA gene restriction patterns provide increased sensitivity for typing Salmonella typhi strains. J Infect Dis 1989;160: 145-9.

\section{Lactobacillus acidophilus Endocarditis After an Appendectomy}

SIR - Lactobacillus endocarditis is a rare infection usually observed only in patients with preexisting structural heart disease who have recently undergone dental work [1,2]. We report an unusual case of native-valve endocarditis due to Lactobacillus acidophilus, with the digestive tract as the probable portal of entry.

A 28-year-old woman was hospitalized in early October 1993 following an 11-week history of fever (temperature, $38.5^{\circ}-$ $39^{\circ} \mathrm{C}$ ), night sweats, and fatigue, and a $7-\mathrm{kg}$ weight loss.

Seven days before the onset of symptoms, the patient had undergone a celioscopic appendectomy. Postoperative followup revealed a previously undetected heart murmur. Transthoracic echocardiograms showed prolapse of the anterior cusp of the mitral valve with no vegetation. One month before admission to the hospital, the patient had complained of severe pain in the left hypochondrium and was given trimethoprim-sulfamethoxazole and pefloxacin for 6 days. She had cramps in her right calf 48 hours before admission. The patient reported that she had no history of valvular heart disease, had not had recent dental work, and did not use intravenous drugs. She did not have an intrauterine contraceptive device.

On admission to the hospital, physical examination revealed a pansystolic murmur in the mitral area, a temperature of $39^{\circ} \mathrm{C}$, splenomegaly, and a tender right calf with moderate edema.

Results of laboratory tests were as follows: erythrocyte sedimentation rate, $60 \mathrm{~mm} / \mathrm{h}$; white blood cell count, $12,600 / \mu \mathrm{L}$ (78\% neutrophils); and circulating immune complexes, $5.6 \mu \mathrm{g} /$ $\mathrm{mL}$ (detected by latex agglutination test). Lupus anticoagulant was not detected.

Transesophageal echocardiograms revealed a large vegetation $(6 \times 10 \mathrm{~mm})$ on the anterior leaflet of the mitral valve that extended into the left ventricle, and a moderate regurgitation. Abdominal echograms showed heterogeneous splenomegaly, suggesting multiple splenic infarcts. A deep venous thrombosis of the right posterior tibial vein was detected by Doppler ultrasonography. No abnormalities were seen on chest, dental, and sinus roentgenograms. Cultures of urine were sterile.

After 12 cultures of blood were set up, empirical intravenous therapy combining penicillin $G$, tobramycin, and heparin was started. Fever still persisted 72 hours later, at which time the

Reprints or correspondence: Dr. Didier Bessis, Service de Médecine Interne A, Hôpital Saint-Eloi, 2, av Bertin-Sans, 34295 Montpellier Cedex 5, France.

Clinical Infectious Diseases 1995;20:724-5

(c) 1995 by The University of Chicago. All rights reserved. $1058-4838 / 95 / 2003-0049 \$ 02.00$ patient developed 10 Osler's nodes in the palmar and plantar areas and multiple asymptomatic maculopapules on the anterior surface of the lower left limb, predominantly around a homolateral effusion in the knee joint that was too small to be aspirated. All blood cultures were positive for organisms within 2-5 days (bioMérieux Vital System; bioMérieux, Marcy l'Etoile, France); L. acidophilus was identified (Bacterial Identification Laboratory, Institut Pasteur, Paris). The strain was susceptible to amoxicillin, ticarcillin, piperacillin, erythromycin, clindamycin, rifampin, and vancomycin, and it was resistant to cefoxitin, gentamicin, tetracycline, trimethoprim-sulfamethoxazole, and metronidazole.

The patient was treated intravenously with a combination of vancomycin and rifampin, and she was apyretic within 48 hours; cutaneous and articular manifestations disappeared within 4 days. The patient then complained of severe pain in the left inguinal fold extending to the anterior thigh. Doppler ultrasonograms showed a partial occlusion of the superficial femoral artery, which was revascularized by Fogarty embolectomy. Pain persisted around the left inguinal scar 14 days after surgery, so arteriography was performed; it revealed a saccular aneurysm $(3.5 \mathrm{~cm}$ in diameter) at the base of the left femoral artery bifurcation. The patient's pain diminished after arterial resection and grafting.

After 6 weeks of intravenous antibiotic therapy, a 15-day course of oral amoxicillin was prescribed. Follow-up cultures of blood taken during antibiotic therapy and after it was discontinued were negative. The vegetation was no longer visible on transthoracic echocardiograms after 8 weeks of antibiotic therapy. The patient was discharged at that time. No recurrence was observed at a follow-up visit 3 months later.

Endocarditis due to Lactobacillus species is rare. Griffiths et al. [2] found only 41 cases reported in the English-language literature since 1938. Lactobacillus species are gram-positive, nonsporulated, anaerobic bacteria that normally reside in the human oral cavity, gastrointestinal tract, and female genital tract. In our patient, recent colon surgery seemed to be the only plausible portal of entry. Indeed, lactobacillus bacteremias originating from the intestinal tract have been described, particularly in patients who have recently undergone endoscopy $[3,4]$. To the best of our knowledge, this is the first report of the development of lactobacillus endocarditis after celioscopic appendectomy. This case suggests that patients with mitral valve prolapse who undergo appendectomy are at risk for developing endocarditis $[5,6]$.

\section{Didier Bessis, Alain Le Quellec, Albert Sotto, Colette Perez,} and Albert-Jean Ciurana

Service de Médecine Interne A, Hôpital Saint-Eloi, Montpellier; Service de Médecine Interne B. Hôpital Caremeau, Nimes; and Laboratoire de Bactériologie. Hôpital Arnaud de Villeneuve, Montpellier, France 